\title{
Citations
}

\section{Silent revolution}

RNA interference has transitioned from a puzzling phenomenon to a routine laboratory tool. But new evidence published by Gullerova and Proudfoot in Nature Structural and Molecular Biology suggests that the traditional method for inducing gene silencing is ripe for a shake-up. Typically, when people refer to RNAi, they are discussing post-transcriptional gene silencing, in which cytoplasmically located mRNA is degraded or prevented from being translated. However, in some organisms, RNAi can occur by transcriptional gene silencing (TGS) via DNA methylation or histone modifications. Like mRNAdirected RNAi, TGS involves processing of double-stranded RNA (dsRNA) into short interfering RNAs that prime a silencing complex. In fission yeast, where TGS is well studied, the dsRNA arises from convergent transcription (in which a DNA sequence is simultaneously transcribed in sense and antisense orientations by promoters at either extreme) and silences in a cis manner, inducing heterochromatin at the very location where it originated. Intriguingly, convergent transcription has been observed to induce gene silencing in mammalian cells as well, but this was assumed to occur post-transcriptionally. In the new work, Gullerova and Proudfoot amass substantial evidence that convergent transcription induces trans TGS in both fission yeast and mammalian cells. The most compelling application in Schizosaccharomyces pombe for the new method is for the manipulation of essential genes, which have historically been studied with temperature-sensitive mutations that necessitate use of nonphysiological temperatures. In the new approach, a plasmid giving inducible convergent transcription of sequences from the target gene is stably transformed. Upon expression, the dsRNA triggers reduced Pol II occupancy and heterochromatin marks in the endogenous gene, leading to transcriptional silencing. For tests in mammalian cells, the authors prepared a plasmid containing two

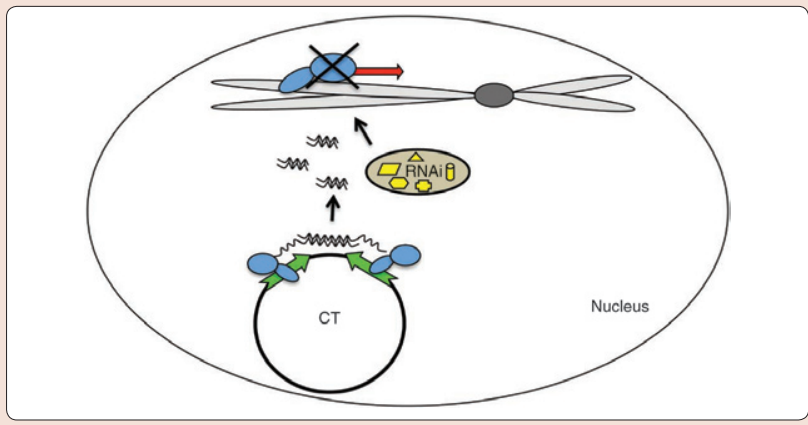

Convergent transcription for transcriptional gene silencing in mammalian cells. Source: Nature Structural and Molecular Biology

opposing cytomegalovirus promoters. Testing confirmed that the profound silencing observed occurred at the chromatin level. The convergent transcription products acted entirely within the nucleus, since no evidence of the dsRNA-induced interferon response was noted, and targeting of intronic and exonic sequences worked comparably. Importantly, transcription-induced TGS outperformed posttranscriptional silencing via siRNA or a hairpin RNA-expressing vector. This work solidifies tantalizing clues suggestive of trans TGS in fission yeast and mammalian cells, and shows how to harness this phenomenon for strong, persistent silencing.

M. Gullerova and N.J. Proudfoot. Convergent transcription induces transcriptional gene silencing in fission yeast and mammalian cells. Nat Struct Mol Biol. [Epub ahead of print, September 30, 2012; doi:10.1038/nsmb.2392].

\section{Guilt by association}

Genome-wide association studies can pinpoint single-nucleotide polymorphisms (SNPs) in linkage disequilibrium with disease. But distinguishing innocent bystander SNPs-mere cosegregating markers-from the true guilty parties is formidable when the base changes occur in noncoding regions. One way a noncoding SNP may exert its effect is by altering binding of transcription factors affecting expression of genes in the vicinity. Allele-specific protein binding has been investigated by chromatin immunoprecipitation, but this approach presupposes which factors will be relevant. On the other hand, an unbiased proteome-wide screen is hampered by the subtle differences in DNA-protein binding affinity when sequences differ only modestly. Now, an article in PLoS Genetics from Butter et al. casts that concern aside by introducing PWAS, or proteome-wide analysis of SNPs. In PWAS, DNA fragments are prepared that contain multiple copies of a given allele in the context of about 40 nucle- otides of surrounding sequence. The DNA concatemers are coupled to beads, and separate aliquots are then incubated with metabolically labeled heavy or light nuclear extract. Meanwhile, the same process is performed for oligonucleotides bearing the other allele. After incubation, the light extract with allele 1 is combined with the heavy extract/ allele 2 sample, and vice versa. Following elution from the beads, the samples are processed for quantitative mass spectrometry. In the absence of allele-specific binding, heavy and light signals for a protein will be of equal intensity. Instances of apparent differential binding are considered artefactual unless they are seen in both pulldowns. This built-in check and the use of concatenated oligonucleotides give the specificity and sensitivity to identify candidate transcription factors that can be verified in functional assays. Showing data from SNPs in the interleukin 2 receptor locus that have been linked to susceptibility to type 1 diabetes, Butter et al. report that they identified biologically plausible proteins, including one, RUNX1, that has already been implicated in other autoimmune diseases and showed allele-specific activation in a reporter gene assay. This test case provides evidence that PWAS will help convert lists of risk alleles into a better functional understanding of disease etiology and pathogenesis.

F. Butter et al. 2012. Proteome-wide analysis of disease-associated SNPs that show allele-specific transcription factor binding. PLoS Genet. 8(9):e1002982.

Selected and written by Nijsje Dorman, Ph.D. [GG]

BioTechniques 53:335 (December 2012)

doi $10.2144 / 000113958$

To purchase reprints of this article, contact: biotechniques@fosterprinting.com 